\title{
Review of Non-Contrast MR-Lymphography Techniques and Findings: Challenges and Opportunities
}

\author{
Abdullah Almujally, Fabrizio Calliada \\ University of Pavia, Radiology Department, Pavia, Italy \\ Email: abdullah.almujally@gmail.com
}

How to cite this paper: Almujally, A. and Calliada, F. (2020) Review of Non-Contrast MR-Lymphography Techniques and Findings: Challenges and Opportunities. Open Journal of Radiology, 10, 35-44. https://doi.org/10.4236/ojrad.2020.102005

Received: February 22, 2020

Accepted: April 21, 2020

Published: April 24, 2020

Copyright $\odot 2020$ by author(s) and Scientific Research Publishing Inc. This work is licensed under the Creative Commons Attribution International License (CC BY 4.0).

http://creativecommons.org/licenses/by/4.0/

\section{(c) (i) Open Access}

\begin{abstract}
Lymphedema is a major disorder of lymphatic system that occurs primarily due to lymphatic system disorders or secondary lymphedema which is caused by radiation therapy involving lymph nodes or surgical operation dissection. Magnetic resonance lymphography (MRL) has been used to effectively examine the lymph vessels and identify their morphology. This review study intended to evaluate the role of non MR-lymphographic for the diagnosis assessment of lymphedema and to provide comprehensive review on clinical outcome of non-contrast MRI compared to MRI with contrast medium. In this study, scientific publications published in languages other than English were excluded. A systematic review was done in international databases including PubMed, Scopus, web of sciences, conference proceedings published till 2019. Non contrast MRI lymphography has strong capability to be the main imaging procedure of choice in the diagnosis lymphedema with accuracy up to $90 \%$.
\end{abstract}

\section{Keywords}

Noncontrast MR Lymphography, Lymphedema, Magnetic Resonance Lymphangiography, Lymphatic Imaging

\section{Introduction}

Lymphatic system disorders are one of the common pathologic conditions that affect a wide range of population worldwide [1]. Lymphatic system, which is a part of circulatory system, has various functions including immunity, fat absorption and fluid homeostasis. Lymphedema or also known as elephantiasis is a lymphatic system chronic disorder caused by accumulation of lymph (protein 
rich fluid), the interstitial space which diminishes lymphatic return, resulting in swelling of one or more regions of the body, is one of the common diseases worldwide [1] [2]. Lymphydema responds dynamically when presented with infection, inflammation, altered hemodynamics, and cancer. Lymphedema symptoms include in addition to swelling, skin atrophy, pain and infection [3] [4]. It was estimated that the incidence of this disease ranges from 140.0 to 250.0 million people globally ( 1 in 30 people) [5] [6] [7]. Lymphedema can affect all body parts and organs but more common in certain organs such as lower limbs, upper limbs with incidence of $99 \%$ of total affected organs or regions [1]. Lymphedema can be classified into primary and secondary types according to the cause of the disease whether it's congenital or acquired. Primary lymphedema is caused by congenital abnormalities (incidence $\approx 1.15$ to 100000 ), whereas secondary lymphedema is caused by infection (filariasis), trauma, surgery, or radiation due to cancer treatment [2] [4]. The causes of lymphedema depend on the economic level of a country. i.e. in developed countries such as the United States and Europe, the lymphedema incidence is 1.4 per 1000 persons, typically secondary due to cancer treatment ( $8 \%$ after mastectomy, $38 \%$ after radiotherapy and axillary lymph node surgical removal) or cancer itself due to blockage of lymph vessels by metastasis. The main cause of lymphedema in developing countries is infection due to the parasite Wuchereria bancrofti (filariasis) in developing countries in southern part of the globe. Furthermore, It was estimated that almost 1.2 billion people are at risk for contraction of lymphatic filariasis in these countries (Figure 1) [8]. Currently, more than 120 million people are affected by lymphatic filariasis. Other risk factors of lymphedema include patient age, body mass index (BMI > 59, obese class III) is high risk for spontaneous development of lower limb lymphedema, extremity infections and trauma, chronic venous insufficiency.

The lymphatic system plays an important role in human health. It composed of huge network of vessels, nodes and lymphatic organs. Diagnostic and therapeutic intervention with various imaging modalities is required to obtain accurate diagnosis. Medical imaging is imperative to for accurate diagnosis with quantitative

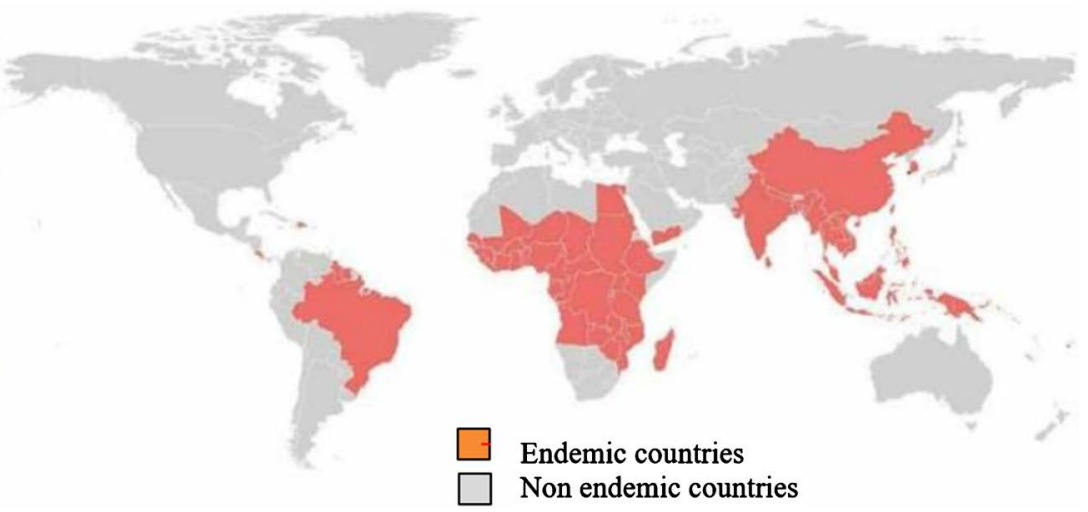

Figure 1. Lymphatic filariasis endemic countries (approximately $18 \%$ of the population worldwide) [8]. 
and qualitative data. However, the lymphatic system is physically complex and challenging to obtain diagnostic image [9]. Non-invasive imaging modalities with no radiation exposure is preferred for repeated procedures for close follow-up of treatment outcome.

Imaging of lymphatic systems (lymphangiography) was first introduced to clinical practice in 1951 using an oil-based contrast medium [10] (Figure 2). The procedure is an invasive, complex and causing patient discomfort and is no longer performed due to its severe complications. Nuclear medicine scintigraphy of the lymph system (Lymphoscintigraphy (LS)) is frequently used for the diagnosis of the system disorders. The main drawback of this technique is the low image resolution and the procedure accompanied of ionizing radiation for patient. More recently, magnetic resonance lymphography (MRL) has been used to effectively examine the lymph vessels and identify their morphology [11] [12]. Different imaging techniques are used to diagnose lymphatic disorders (lymphangiography, lymphoscintigraphy, computed tomography (CT), MRL, positron emission tomography (PET)/CT and ultrasound imaging). Non-invasive measurements are also used for diagnosis of lymphedema such as tonometry (tissue resistance assessment), bioimpedence analysis spectroscopy (extracellular fluid assessment), fluorescence imaging and near-infrared fluorescence (NIRF) lymphatic imaging may be useful in early onset of the disease to obtain accurate diagnosis [2]. However, all these techniques have different limitations [13]. Contrast agent is used to create informative radiographic images by direct or indirect injection of contrast in the vessel. Direct injection may induce sever complications, which lead to elimination of this technique. On the other hand, indirect injection conducted by injection the contrast interstitially. Other techniques were developed to overcome the limitation of conventional ones. These techniques include scintigraphy, PET, single photon emission computed tomography (SPECT), CT and

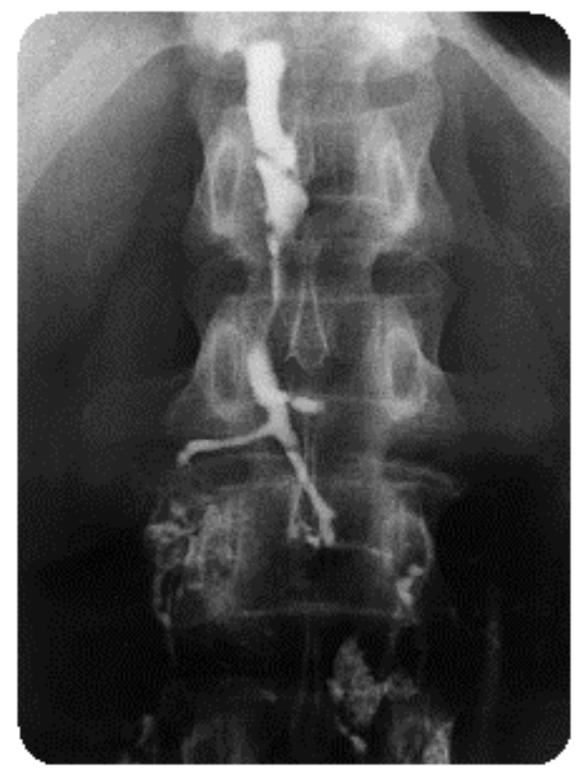

Figure 2. lymphogram of the thoracic canal [9]. 
MRI. Currently, MRI and PET MRI are the best imaging modalities to assess the pathology. Contrast MRI is used usually to alter relaxation time (Shorten the T1 or T2 relaxation time, thereby causing increased signal intensity on T1-weighted images or reduce signal intensity on T2-weighted images) to enable accurate visualization of the anatomy and pathology (gadolinium compounds) [13] [14]. However, MRI contrast media has side effects that can be classified into three categories minor (nausea, vomiting, sneezing), mild (persistent headache, hypotension with bradycardia and severe side effects (Laryngeal edema and cardiac arrest) according to the patients and contrast volume and contrast type. Non contrast MRL is promising technique that provides accurate diagnosis without contrast medium, thus protect patients from these adverse side effects. Furthermore, non-contrast MRL is an emerging technique for noninvasive diagnosis of Lymphedema and limited literature is available in this field. Therefore, we conducted this study to evaluate the existing scientific literature to evaluate whether non-contrast MRL is capable to provide precise and accurate diagnosis of lymphedema. This review study intended to evaluate the role of non MR-lymphographic for the diagnosis assessment of lymphedema and to provide comprehensive review on clinical outcome of non-contrast MRI compared to MRI with contrast medium.

\section{Materials and Methods}

In this study included scientific articles published in English languages journals regarding non contrast MRI for diagnosis lymphedema. A systematic review was done in international databases including PubMed, Scopus, web of sciences, conference proceedings published till 2019.

\section{MR lymphographic Imaging}

Early diagnosis positive affect the treatment outcome which results in prompt progress of treatment and may prevent complications such as secondary fibrosis [15]. It is well documented that MRL provides useful anatomical and physiological data, thus enabling accurate diagnosis of the disease. It was reported that the sensitively and specifity of the procedure is $90 \%$ and $94 \%$ respectively [16]. Many authors reported the advantages of contrast studies and others attempt to acquire images without contrast medium [11] [13] [14] [16]. (Table 1) MRL consists of two sequences. The first is a $3 \mathrm{D}$ heavily $\mathrm{T} 2$-weighted sequence to depict the severity and extent of the lymphedema. The second is a fat-suppressed 3D spoiled gradient-echo sequence performed after the intra-cutaneous injection of an extracellular gadolinium-based MR contrast agent [16]. In addition to that, the authors reported that as venous enhancement almost always occurs, one of the interpretative challenges is differentiating enhancing lymphatic channels from superficial veins. Magnetic resonance imaging (MRI), which is chemically sensitive in-vivo imaging technique, is a medical noninvasive imaging technique that produce a three dimensional (3D) images of human body using magnet and 
Table 1. Advantages and drawbacks of various imaging modalities.

\begin{tabular}{|c|c|c|}
\hline Imaging modality & Drawbacks & Advantages \\
\hline Lymphangiography & $\begin{array}{l}\text { Fever, Infection and lymph vessels } \\
\text { inflammation, contrast medium } \\
\text { remain for up to } 2 \text { years in lymph } \\
\text { nodes }\end{array}$ & $\begin{array}{l}\text { Demonstrate disorders of the } \\
\text { internal tissues of lymph } \\
\text { nodes }\end{array}$ \\
\hline Lymphoscintigraphy & $\begin{array}{l}\text { Low image resolution, prolonged } \\
\text { procedure time cost, radiation dose }\end{array}$ & Lymphatic function \\
\hline $\begin{array}{l}\text { Indo cyanine } \\
\text { green-lymphography (ICG-L) }\end{array}$ & $\begin{array}{l}\text { Cannot detect deep lymphatic } \\
\text { vessels }(\leq 2 \mathrm{~cm})\end{array}$ & $\begin{array}{l}\text { insignificantly invasive, simple, } \\
\text { Real-time imaging and } \\
\text { sensitive }\end{array}$ \\
\hline Computed Tomography & $\begin{array}{l}\text { Limited contrast resolution } \\
\text { Radiation risk }\end{array}$ & Cheap, low cost \\
\hline $\begin{array}{l}\text { Magnetic resonance } \\
\text { lymphangiography (MRL) }\end{array}$ & $\begin{array}{l}\text { Contrast medium can not } \\
\text { differentiate benign and cancer } \\
\text { tumors }\end{array}$ & $\begin{array}{l}\text { Excellent anatomy high } \\
\text { spatial resolution No } \\
\text { radiation exposure 3D image } \\
\text { physiological information } \\
\text { high soft tissue contrast }\end{array}$ \\
\hline $\begin{array}{l}\text { Non-contrast Magnetic } \\
\text { Resonance Lymphangiography } \\
\text { (NCMRL) }\end{array}$ & $\begin{array}{l}\text { Suspension the lymphatic drainage } \\
\text { for } 48 \mathrm{~h} \text {. Elastic stockings or } \\
\text { bandages for } 24 \mathrm{~h}\end{array}$ & $\begin{array}{l}\text { No contract medium illustrate } \\
\text { static fluid-filled lymphatic } \\
\text { vessels, safe and feasible }\end{array}$ \\
\hline
\end{tabular}

magnetic characteristics of human body tissues and organs. Since its introduction to clinical use in 1977 [16] MRI systems developed very rapidly to meet the requirements of high diagnostic yield with minimal discomfort and short time.

\section{MRI Sequences and Equipment Setting}

The preferred MR equipment includes a 1.5-Tesla or more MR unit. In our experience, all MR examinations were performed by a General Electric Healthcare Signa Twin Speed HDxt, with a maximum gradient strength value of $23 \mathrm{mT} / \mathrm{m}$ and a slew rate of $80 \mathrm{mT} / \mathrm{m} / \mathrm{ms}$ (software release 15.0-0947A). A multi element body coil is fundamental for this type of examination. For our purposes, we used a receiving phased-array peripheral vascular coil for the study of the lower extremities (Flow 7000 phased-array peripheral vascular, USA Instruments) and an 8-channel body array coil for the upper extremities, with both a large anatomical coverage and a good signal-to-noise ratio.

\section{MR Lymphographic Imaging}

Noncontrast MRL has substantial benefits compared to other imaging techniques. In this context, Arrive et al. in their recent studies [17] [18] and Derhy et al. [19] reported that Noncontrast MRL can be performed using heavily T2weighted fast spin echo sequences (to demonstrate the location of edema) with a long T2 relaxation time. Derhy et al. [19] et al. stated that 3D MRL demonstrates that retroperitoneal lymphatic aneurysmal dilatation exhibits a continuous spectrum of change from normal variants to lymphatic aneurysmal dilatation. The authors reported that this technique was useful to diagnose many clinical 
condition. In another recent study, Arrivé et al. [18] used noncontrast MRL for evaluation of lymph node for Secondary upper limb lymphedema. The authors used a free-breathing three-dimensional fast spin-echo sequence evaluate the results of axillary lymph node transplantation with noncontrast MRL in 15 patients with secondary upper limb lymphedema. The study recommended that Noncontrast magnetic resonance lymphography may be used as an objective technique to analyze the results of lymph node before and after surgery. MR lymphography with very heavily $\mathrm{T} 2$-weighted MR images provides an excellent analysis of both lymphatics vessels and lymph nodes without need of any contrast media. Kim et al. [20] assessed the thoracic duct using coronal and axial images of heavily T2-weighted sequences without contrast medium. Nonenhanced MR lymphangiography is a safe and effective method for imaging the central lymphatic system, and can contribute to differential diagnosis and appropriate preoperative evaluation of pathologic lymphatic problems. The main limitations to noncontrast T2 imaging are that it does not provide information about lymphatic flow and the lack of a contrast medium makes it difficult to visualize small lymphatic ducts. Consequently, its use in diagnostic and interventional lymphangiography is limited [19]. Recently, Cellina et al. [1] reviewed a non-contrast magnetic resonance lymphangiography as an emerging technique for the study of lymphedema. The authors concluded that non-contrast magnetic resonance lymphangiography, a relatively new technique for the diagnosis, assessment and management of lymphedema. Cellina et al. [1] reported that non contrast is useful for the lymphography evaluation and surgical arrangement and provides morphological information regarding size, site of lymph nodes thus can be used for nodal transplantation.

\subsection{Non-Contrast MRL Technique}

\subsubsection{Patient Preparation and Positioning}

MRL without contrast can be performed using MRI system with magnet strength 1.5 or $3.0 \mathrm{~T}$. Before positioning, it is important to explain in detail the diagnostic procedure and the need to maintain the position throughout the duration of the examination. According to the patient's size, the acquisition is usually performed in 3 or 4 steps to cover all the anatomical stations for both lower and upper extremities. In literature, the acquisition techniques described in the literature have provided for the acquisition of the entire limb also in secondary lymphedema, to accurately define the extent and severity of the disorder and to perform an optimal post-treatment follow-up.

\subsubsection{Image Acquisition Protocol}

Non contrast MRL technique is still evolving, but the principle of this examination is based on heavily T2-weighted sequences, resulting in a signal loss in tissue background, with highlighting of static fluids in lymphatic vessels with a very long TR/TE, similar to that used in cholangiopancreatography or in MR urography. Acquisition parameters of non-contrast T2 MR Lymphography se- 
quences according to different authors. Arrivé et al. [18], applied at the end of the echo train a restore pulse to flip the transverse magnetization to the longitudinal direction to reduce the acquisition time. Other sequences can be associated in the examination: axial HASTE without fat suppression (TR: $1200 \mathrm{~ms}$, TE: 114 ms, matrix: $176 \times 256$, flip angle: $180^{\circ}$, slice thickness: $6 \mathrm{~mm}$ ) or Iterative Decomposition of water and fat with Echo asymmetry and Least-squares estimation (IDEAL; acquisition plane: axial, TR: $4233 \mathrm{~ms}$, TE: $76 \mathrm{~ms}$, slice thickness: $6 \mathrm{~mm}$, matrix: $320 \times 192$, FOV: $380 \times 380$ ), this sequence, based on fat/water separation technique, provides contrasts of water and fat, in phase and out of phase and is used for the analysis of lymphedema characteristics [17] [18] [21]-[27] (Table 2).

\section{NCMRL Technique Limitations}

Because lymphedema is common in obese patients, therefore obese patients

Table 2. Image acquisition protocols for MRL in Literature.

\begin{tabular}{|c|c|c|c|c|}
\hline Author & Country & No of Patients & Organ of interest & Protocol \\
\hline Young et al. [6] & Korea & 10 & Thoracic ducts & 3T MR system (MAGNETOM Skyra, Siemens) \\
\hline Xiong et al. $[13]$ & China & Review & All & Lymphoscintigraphy, Lymphangiography SPECT/CT, SPECT, US, MRL \\
\hline $\begin{array}{l}\text { Mitsumori et al. } \\
\qquad[16]\end{array}$ & USA & NA & $\begin{array}{c}\text { Peripheral } \\
\text { lymphedema }\end{array}$ & $\begin{array}{l}\mathrm{GE}, 1.5 \mathrm{~T} \text { or } 3.0 \mathrm{~T} \text {, (Gd-BOPTA), Multi Hance The MR examination consists } \\
\text { of } 2 \text { primary sequences. The first is a } 3 \mathrm{D} \text { heavily T2-weighted sequence }\end{array}$ \\
\hline $\begin{array}{l}\text { Chavhan et al. } \\
\qquad[21]\end{array}$ & USA & Review & MRL & $\begin{array}{l}\text { gadolinium-based contrast material T2-weighted imaging plays a } \\
\text { complementary role to DCE MR lymphangiography in the assessment of } \\
\text { CCLs }\end{array}$ \\
\hline Lu et al. [22] & China & 37 & $\begin{array}{l}\text { Male Genital } \\
\text { Lymphedema }\end{array}$ & $\begin{array}{l}\text { Preoperative and postoperative MRL Philips } 3.0-\mathrm{T}, \mathrm{Gd}-\mathrm{BOPTA} .3 \mathrm{D} \text { fast } \\
\text { spoiled gradient recalled echo T1-weighted images with a fat saturation } \\
\text { technique(TR/TE: } 3.5 / 1.7 \text {, flip angle: } 25\end{array}$ \\
\hline White et al. [23] & UK & NA & $\begin{array}{l}\text { Lower limb } \\
\text { lymphoedema }\end{array}$ & $\begin{array}{l}\text { T Siemens, T2-weighted sequences and coronal FLASH (fast low-angles hot) } \\
\text { T1-weighted spoiled gradient-echo sequences }\end{array}$ \\
\hline Lu et al. [24] & China & 10 & whole-body & $\begin{array}{l}\text { For MR lymphangiography, a 3D THRIVE sequence (T1 high-resolution } \\
\text { isotropic volume excitation, THRIVE) was performed. Pre- and post-contrast } \\
\text { injection was done using the following parameters: TR/TE } 23 / 2.1 \mathrm{~ms} \text {; Flip } \\
\text { angle } 15^{\circ} \text {; FOV } 38 \times 36 \mathrm{~cm}^{2} \text {; matrix size } 760 \times 720\end{array}$ \\
\hline Dori et al. [25] & USA & NA & Animal & $\begin{array}{l}\text { T2-weighted MR sequence, bilateral inguinal lymph node injection of } 2 \mathrm{~mL} \\
\text { of undiluted gadopentetate at a rate of } 1 \mathrm{~mL} / \mathrm{min} \text {, and } 60 \text { minutes of } \mathrm{MR} \\
\text { imaging with T1-weighted high-spatial- and high-temporal-resolution } \mathrm{MR} \\
\text { angiography }\end{array}$ \\
\hline Chen et al. [27] & China & 19 & Thorax & $\begin{array}{l}\text { 3.0T MRI system with the imaging sequence of magnetic resonance } \\
\text { cholangiopancreatography. TD MRL images were obtained from subjects } \\
\text { following an overnight fast and from the same subjects } 3-4 \mathrm{~h} \text { after ingestion } \\
\text { of a high fat meal }\end{array}$ \\
\hline
\end{tabular}


grade three cannot examine in close magnet bore due to their weight and non-contrast MRI is usually performed on $1.5 \mathrm{~T}$ magnet or above [1]. Therefore, open magnet cannot be used which one of the limitations of this technique.

\section{Conclusion}

Early Lymphedema stages diagnosis represents great challenges. Non contrast MRL is used to diagnose accurately the lymphatic system disorder. From studies, the researchers have found non contrast MRL is a promising methodology in the diagnosis of lymphatic system disorders with accuracy up to $90 \%$. The study revealed that non-contrast MRL imaging technique can increase the accuracy of lymphedema diagnosis, improve disease prognostication, and provide a more robust marker of treatment response.

\section{Acknowledgements}

The authors would like to express their sincere appreciation to everyone in the Lymph Team: IRCCS Policlinico San Matteo (PV) at the Department of Radiology, University of Pavia.

\section{Conflicts of Interest}

The authors declare no conflicts of interest regarding the publication of this paper.

\section{References}

[1] Cellina, M., Oliva, G., Menozzi, A., Soresina, M., Martinenghi, C. and Gibellid, D. (2019) Non-Contrast Magnetic Resonance Lymphangiography: An Emerging Technique for the Study of Lymphedema. Clinical Imaging, 53, 126-133. https://doi.org/10.1016/j.clinimag.2018.10.006

[2] O’Donnell Jr., T., Rasmussen, J. and Sevick-Muraca, E. (2017) New Diagnostic Modalities in the Evaluation of Lymphedema. Journal of Vascular Surgery: Venous and Lymphatic Disorders, 5, 261-273. https://doi.org/10.1016/j.jvsv.2016.10.083

[3] Basta, M.N., Gao, L.L. and Wu, L.C. (2014) Operative Treatment of Peripheral Lymphedema: A Systematic Meta-Analysis of the Efficacy and Safety of Lymphovenous Microsurgery and Tissue Transplantation. Plastic and Reconstructive Surgery, 133, 905-913. https://doi.org/10.1097/PRS.0000000000000010

[4] Kayıran, O., De La Cruz, C., Tane, K. and Soran, A. (2017) Lymphedema: From Diagnosis to Treatment. Turkish Journal of Surgery, 33, 51-57. https://doi.org/10.5152/turkjsurg.2017.3870

[5] Mendoza, N., Lia, A, Gill, A. and Tyring, S. (2009) Filariasis: Diagnosis and Treatment. Dermatologic Therapy, 22, 475-490. https://doi.org/10.1111/j.1529-8019.2009.01271.x

[6] Young. K.E., Sup, P.J, Ho, L.K. and Young, H.S. (2016) Incidence and Risk Factors of Lower Extremity Lymphedema after Gynecologic Surgery in Ovarian Cancer. International Journal of Gynecological Cancer, 26, 1327-1332. https://doi.org/10.1097/IGC.0000000000000757

[7] Slavin, S.A, Greene, A.K. and Borud, L.J. (2009) Lymphedema. 2010. In: Weinzweig, 
J., Ed., Plastic Surgery Secrets Plus, 2nd Edition, Mosby, Philadelphia, PA. https://doi.org/10.1016/B978-0-323-03470-8.00096-X

[8] World Health Organization (2014) Featured Map: World: Distribution of Lymphatic Filariasis and Status of Preventive Chemotherapy in Endemic Countries, 2014.

[9] Guermazi, A., Brice, P., Hennequin, C. and Sarfati, E. (2003) Lymphography: An Old Technique Retains Its Usefulness. RadioGraphics, 23, 1541-1560. https://doi.org/10.1148/rg.236035704

[10] Gough, M.H. (1964) Lymphangiography in Children. Archive of Disease in Childhood, 39, 177-181. https://doi.org/10.1136/adc.39.204.177

[11] Carrasco,V., Carreto,A., Garcia-Tutor, E., de la Fuente, E., López, A., Alonso-Burgos, A., et al. (2015) MR-Lymphography: Technique, Indications and Results. European Society of Radiology (ESR), 1-22.

[12] Mazzei, F.G., Gentili, F., Guerrini, S., Cioffi Squitieri, N., Guerrieri, D., Gennaro, P., Scialpi, M., Volterrani, L. and Mazzei, M.A. (2017) MR Lymphangiography: A Practical Guide to Perform It and a Brief Review of the Literature from a Technical Point of View. BioMed Research International, 2017, Article ID: 2598358. https://doi.org/10.1155/2017/2598358

[13] Xiong, L., et al. (2014). Current Techniques for Lymphatic Imaging: State of the Art and Future Perspectives. European Journal of Surgical Oncology, 40, 270-276. https://doi.org/10.1016/j.ejso.2013.11.027

[14] Wu, L., et al. (2011) Diagnostic Performance of USPIO-Enhanced MRI for LymphNode Metastases in Different Body Regions: A Meta-Analysis. European Journal of Radiology, 80, 582-589. https://doi.org/10.1016/j.ejrad.2009.11.027

[15] Szuba, A., Shin, W.S., Strauss, H.W. and Rockson, S. (2003) The Third Circulation: Radionuclide Lymphoscintigraphy in the Evaluation of Lymphedema. Journal of Nuclear Medicine, 44, 43-57.

[16] Mitsumori, L.M., McDonald, E.S., Neligan, P.C. and Maki, J.H. (2016) Peripheral Magnetic Resonance Lymphangiography: Techniques and Applications. Techniques in Vascular Interventional Radiology, 19, 262-272. https://doi.org/10.1053/j.tvir.2016.10.007

[17] Arrivé, L., Derhy, S., El Mouhadi, S., Monnier-Cholley, L., Menu, Y. and Becker, C. (2016) Noncontrast Magnetic Resonance Lymphography. Journal of Reconstructive Microsurgery, 32, 80-86. https://doi.org/10.1055/s-0035-1549133

[18] Arrivé, L., Derhy, S., Dlimi, C., El Mouhadi, S., Monnier-Cholley, L. and Becker, C. (2017) Noncontrast Magnetic Resonance Lymphography for Evaluation of Lymph Node Transfer for Secondary Upper Limb Lymphedema. Journal of Reconstructive Microsurgery, 140, 806e-811e. https://doi.org/10.1097/PRS.0000000000003862

[19] Derhy, S., El Mouhadi, S., Ruiz, A., Azizi, L., Menu, Y. and Arrivé, L. (2013) Non-Contrast 3D MR Lymphography of Retroperitoneal Lymphatic Aneurysmal Dilatation: A Continuous Spectrum of Change from Normal Variants to Cystic Lymphangioma. Insights Imaging, 4, 753-758. https://doi.org/10.1007/s13244-013-0290-4

[20] Kim, E.Y., Hwang, H.S., Lee, H.Y., Cho, J.H., Kim, H.K., Lee, K.S., Shim, Y.M. and Zo, J. (2016) Anatomic and Functional Evaluation of Central Lymphatics with Noninvasive Magnetic Resonance Lymphangiography. Medicine, 95, e3109. https://doi.org/10.1097/MD.0000000000003109

[21] Chavhan, G.B., Amaral, J.G., Temple, M. and Itkin, M. (2017) MR Lymphangiogra- 
phy in Children: Technique and Potential Applications. RadioGraphics, 37, 1775-1790. https://doi.org/10.1148/rg.2017170014

[22] Lu, Q., Jiang, Z., Zhao, Z., Wu, L., Wu, G., Suo, S. and Xu, J. (2016) Assessment of the Lymphatic System of the Genitalia Using Magnetic Resonance Lymphography Before and After Treatment of Male Genital Lymphedema. Medicine (Baltimore), 95, e3755. https://doi.org/10.1097/MD.0000000000003755

[23] White, R.D., Weir-Mccall, J.R., Budak, M.J., Waugh, S.A., Munnoch, D.A. and Sudarshan, T.A.P. (2014) Contrast-Enhanced Magnetic Resonance Lymphography in the Assessment of Lower Limb Lymphedema. Clinical Radiology, 69, e435-e444. https://doi.org/10.1016/j.crad.2014.06.007

[24] Lu, Q., Bui, D., Liu, N.F., Xu, J.R., Zhao, X.H. and Zhang, X.F. (2012) Magnetic Resonance Lymphography at 3T: A Promising Noninvasive Approach to Characterise Inguinal Lymphatic Vessel Leakage. European Journal of Vascular and Endovascular Surgery, 43, 106-111. https://doi.org/10.1016/j.ejvs.2011.09.007

[25] Dori, Y., Zviman, M. and Itkin, M. (2014) Dynamic Contrast-Enhanced MR Lymphangiography: Feasibility Study in Swine. Radiology, 273, 410-416. https://doi.org/10.1148/radiol.14132616

[26] Liu, N., Wang, C. and Sun, M. (2005) Noncontrast Three-Dimensional Magnetic Resonance Imaging vs Lymphoscintigraphy in the Evaluation of Lymph Circulation Disorders: A Comparative Study. Journal of Vascular Surgery, 41, 69-75. https://doi.org/10.1016/j.jvs.2004.11.013

[27] Chen, S., Tan, X., Wu, R., Xu, Y., Yang, C., Wang, M., Liu, F., Wang, Z. and Yuan, K. (2017) Non-Enhanced MR Lymphography of the Thoracic Duct: Improved Visualization Following Ingestion of a High Fat Meal-Initial Experience. Clinical Physiology and Functional Imaging, 37, 730-733. https://doi.org/10.1111/cpf.12366 